\title{
笑傲江湖之金属有机骨架材料
}

杨祥，韩爱娟“，刘军枫

北京化工大学化学学院, 化工资源有效利用国家重点实验室, 北京 100029

摘要：金属有机骨架材料(MOFs)是一种具有三维周期性网状结构的化合物。由于 MOFs 具有孔隙率高、比表面积 大和孔径可调节等特点, 在吸附分离方面表现出极佳的性能。本文运用拟人化的手法, 向读者展示 MOFs 的性质、 结构调控和在吸附分离方面的应用。

关键词: MOFs; 气体吸附; 液相吸附

中图分类号: G64; O6

\section{The Legendary Swordsman: MOFs}

\section{Xiang Yang, Aijuan Han *, Junfeng Liu}

State Key Laboratory of Chemical Resource Engineering, College of Chemistry, Beijing University of Chemical Technology, Beijing 100029, China.

Abstract: Metal-organic frameworks (MOFs) are a kind of compounds with a three-dimensional periodic network structure. MOFs exhibit excellent performance in adsorption and separation due to their high porosity, large specific surface area and adjustable pore size. This paper uses the anthropomorphic technique to show the nature, structure regulation as well as adsorption and separation applications of MOFs to the readers.

Key Words: MOFs; Gas adsorption; Liquid phase adsorption

MOFs作为一种新兴的多孔纳米材料, 面对江湖中的活性炭、树脂等各门派可谓是初生牛犊不怕 虎, 尤其是在吸附领域已经占据一席之地。MOFs到底修炼了什么技能可以让他在短短20多年的时间 内大展身手? 下面让我们一起穿梭于江湖中, 探索其中的奥秘吧。

\section{1 骨骼精奇 天赋异禀}

首先, 我们来到了美国加州伯克利分校, MOFs的元老MOF-5 ${ }^{[1]}$ 就是在这里诞生的。据说在Yaghi 教授的 “炼丹炉” 中, MOF-5可是经历了高温高压等重重险阻, 通过有机配体对苯二甲酸与金属锌 离子构筑而成。特有的多孔结构赐予了他巨大的比表面积(高达 $2900 \mathrm{~m}^{2} \cdot \mathrm{g}^{-1}$ ), 如果他把体内 $1 \mathrm{~g}$ 的骨 架推平的话会占据 1 个标准的足球场, 其比表面积超过了当时所有已报道的多孔材料。MOF-5也是不 知谦虚, 听到有人在夸赞他, 就迫不及待地向我们展示他那金刚不坏之身 (热稳定性高达 $\left.300{ }^{\circ} \mathrm{C}\right)$ 。原 来他的结构是每 4 个锌原子构成 1 个金属节点, 被刚性配体对苯二甲酸的氧原子固定连接成立方体结 构。

下一站, 我们又来到了挪威奥斯陆大学。著名的 $\mathrm{UiO}$ 系列是 $\mathrm{MOF} 门$ 门派的一个分支, 在江湖中是 
如雷贯耳, 以所在地University of Oslo命名。其中UiO-66是由金属Zr与对苯二甲酸中的氧原子配位而 成 ${ }^{[2]}$, 热稳定性高达 $540^{\circ} \mathrm{C}$ 。与MOF-5不同的是，每六个锆原子构成一个金属节点, 在对苯二甲酸的 连接下构筑成八面体结构。由于金属节点和有机配体选择的多样性使这一门派人才济济, 单是从结 构划分MOFs的种类在2013年底就已经达到了 20000 种 ${ }^{[3]}$, 我们还是一起看看一些著名分支吧(表1)。

表1 MOFs的命名规则及几种高性能的MOFs

\begin{tabular}{ccc}
\hline 名称 & 命名规则 & 代表 \\
\hline MIL- $x$ & 以发现单位Materials of Institute Lavoisier首字母命名 & MIL-101 ${ }^{[4]}$ \\
ZIF- $x$ & 结构类似沸石, 以Zeolitic Imidazolate Framework命名 & ZIF-8 ${ }^{[5]}$ \\
HKUST- $x$ & 以发现单位Hong Kong University of Science and Technology首字母命名 & HKUST-1 ${ }^{[6]}$ \\
NU- $x$ & 以发现单位Northwestern University首字母命名 & NU-110 ${ }^{[7]}$ \\
\hline
\end{tabular}

\section{2 吸星大法 傲视群雄}

吸星大法是武林中绝学, 须令丹田 “常如空箱, 恒似深谷”, 以空洞的方式吸附他人内力。而 MOFs 与生俱来的多孔结构提供了足够多的孔洞用于吸附储存内力, 从一出生就习得了这吸星大法。

听说最近地球计划进入 “氢经济” 时代, 而氢经济主要由氢制取、氢储存和氢应用所构成, MOFs 打算先在氢能储运这一方面大展身手。目前, 吸附储氢的任务主要由碳纳米管和活性炭等碳基门派 包揽, MOFs 首先派出了MOF-5 作为代表去挑战擂台 ${ }^{[8]}$ 。MOF-5 在这些前辈面前可谓是 “长江后浪推 前浪”, 只见他与氢同时处在 $78 \mathrm{~K}, 7 \times 10^{4} \mathrm{~Pa}$ 环境内, 深吸一口气, 运用吸星大法将氢分子吸附到自 身的孔道内, 储氢量经裁判计算达到了 $4.5 \%(w)$ 。台下的观众一片哗然, 只见Tibbetts教授提出质疑: “储氢密度受操作条件的影响比较大, 在 2001 年我就说过, 没有碳材料可以在室温下储氢量达到 $1.0 \%{ }^{[9]}$, 你敢不敢挑战一下? ” MOF- 5 明知这是激将法, 但他还是接受了挑战。在众人注目下, MOF5 的储氢量最后不多不少, 刚好 $1.0 \%$ ！赢得了台下观众的一片掌声。

原来, MOF-5在来之前就已经做好了足够的功课, 其实自己和其他碳基材料类似, 都是多孔结 构, 唯一不同的是自身骨架内含有不饱和的金属位点。那么不饱和金属位点是怎么来的呢? 在吸附 氢过程中又起着怎么样的作用呢? 这就要追溯到MOF-5在 “炼丹炉” 内的生长过程了, 由于炉内含 有二甲基甲酰胺、水等溶剂小分子, 且金属中心也存在 “七情六欲” , 找不到对苯二甲酸进行配位, 只能寻找这些小分子进行结合满足自己的配位需求。在使用吸星大法之前, MOF-5需要在真空环境 下承受高温磨练, 这些溶剂分子就离开了金属中心, 最后在骨架内留下了孤单的不饱和金属位点。在 储氢过程中, 这些不饱和金属位点起到了重要的作用, 因为金属与氢之间的吸附力强于碳与氢, 所 以MOF-5才会比传统的碳基门派具有更强大的储氢能力。继MOF-5挑战擂台之后, 一大批MOFs 开始 登上储氢的舞台, 因为MOFs结构可调、可掺杂金属等特点, 储氢量记录也在不断地刷新。

\section{3 珠联璧合 相得益彰}

正所谓 “时势造英雄” , 英雄的出现绝非偶然。近年来, 碳中和问题在全球掀起一阵热潮。作 为碳中和的关键步骤, 碳捕集与封存技术发挥着关键的作用。这次MOFs可不再单枪匹马了, 自从上 次MOFs和碳基材料两大门派在储氢量上针锋相对后, 两大家子也算是不打不相识, 正准备一起合作 报名参加 $\mathrm{CO}_{2}$ 吸附比赛呢。考虑到氧化石墨烯(Graphene Oxide, GO)具有层间通道分离效应, 碳基材 料任命 $\mathrm{GO}$ 为代表, 同UiO-66前往擂台 ${ }^{[10]}$ 。

“现在我宣布比赛规则, 因为在 $\mathrm{CO}_{2}$ 中混有其他气体分子, 各位选手如果没有对混合气体中 $\mathrm{CO}_{2}$ 进行选择性吸附分离, 则被淘汰。”主持人刚宣布完, 就看到这兄弟俩身披黄色 “战袍” 携手登上 擂台。在炼丹炉中, UiO-66早已练得 “火眼金睛” , 使用吸星大法将 $\mathrm{CO}_{2}$ 牢牢地吸附到自身空腔内。 $\mathrm{GO}$ 也不甘示弱, 在比赛前将自身层间距调整到 $\mathrm{CO}_{2}$ 和 $\mathrm{N}_{2}$ 分子直径之间, 且体内富含 $-\mathrm{COOH}$ 和 $-\mathrm{OH}$ 
等极性基团使得 $\mathrm{CO}_{2}$ 顺着层间通道快速吸附, 而 $\mathrm{N}_{2}$ 还在哭丧着脸被拒之门外。一些 $\mathrm{N}_{2}$ 分子趁着两人 正专心施法, 打算偷偷从他们身旁绕过去, 没成想又被黄色 “战袍” 阻挡住了, 这下 $\mathrm{N}_{2}$ 可真是没辙 了。台下的观众一片叫好, 但是心里都泛着嘀咕: 这火眼金睛和黄色战袍是哪里来的呢?

原来, $\mathrm{UiO}-66$ 在途中听说乙醇胺溶液在 $\mathrm{CO}_{2}$ 吸附中具有举足轻重的地位, 便急忙前去拜访。得知 他来意后, 乙醇胺也毫不吝啬地助他 “一臂之力” 。即赋予他具有可以提供电子的官能团一 $\mathrm{NH}_{2}$, 在 $\mathrm{CO}_{2} / \mathrm{N}_{2}$ 中对二氧化碳具有较强的亲和力。且氮是一种带负电性的原子, 可作为捕获 $\mathrm{CO}_{2}$ 的活性位点。 在乙醇胺前辈提供的思路下, $\mathrm{UiO}-66$ 可是如虎添翼了。武林中人集思广益, 将有机配体替换为2-氨 基对苯二甲酸, 让 $\mathrm{UiO}-66$ 打一出生就带有 $-\mathrm{NH}_{2}$ 。而黄色战袍其实是高分子膜派来协助二人的聚亚 酰胺膜, 在分离过程中起到了选择性屏障的作用。通过这次三大门派的合作, 各种金属氧化物也顺 应发展潮流与MOFs建立起良好的合作关系, 将各自的性能发挥到极致, 真可谓是 “珠联璧合, 相得 益彰”啊!

\section{4 天降大任 劳其筋骨}

MOFs门派在气相吸附中已经站稳了脚跟, 在储氢和天然气储存等比赛中崭露头角后, 还联合各 大门派解决了碳捕集和轻烃分离等问题, 在武林中傲视群雄。这次, MOFs向众人承诺要挂帅出征, 踏上液相吸附的征途, 争取一鸣惊人。

与气相吸附不同, 液相吸附不仅要求MOFs高比表面积和高孔隙率, 还要求MOFs能在水中处之 泰然。为了准备出征, 一些MOFs将自己闭关于水中修炼, 本以为会像之前在炼丹炉内可练成金刚不 坏之身, 可是过了几天发现自身内部骨架正在坞塌, 最终和水融为一体。出师未捷身先死, 长使英 雄泪满襟。悲痛之余, MOFs门派通过这次失败的教训也认识到自身的不足, 原来是金属与配体配位 键较弱, 导致MOFs长时间接触水使结构发生分解。为了解决这个问题, MOFs内部召开会议商讨新 的方针。

会上军师沉默良久, 仰天笑道: “善将者以柔克刚, 硬碰硬不行, 我们可以采用迂回策略。倘 若大家在配体中引入疏水基团或者在自身表面覆盖疏水层, 那么大家对水不就避而远之了! 液相吸 附之路, 我们势在必行。”

MIL-101怒斥军师: “你不用高兴得这么早, 我们是去吸附物质的, 不是游泳划水的, 如何进一 步提高对物质的吸附量才是关键。”

军师并没有与他辩论, 只留下一句孟子的名言便离开了: “天降大任与斯人也, 必先苦其心志, 劳其筋骨, 饿其体肤, 空乏其身, 行拂乱其所为。”

MIL-101对军师所言字字煁酌, 突然恍然大悟, 明白了 “苦其心志, 劳其筋骨” 的含义。因为MIL101-Cr在形成的初期存在爆发成核的现象, 导致颗粒内层存在缺陷。利用醋酸提供的质子与配体上 的氧形成氢键, 内层不稳定的骨架结构会被优先刻蚀, 只留下颗粒外层形成空心球。空心结构的形 成为吸星大法提供了足够多的 “空洞”, 吸附量自然提升了。但是空心球还存在一个问题, 如果壳 层太薄, 相当于鸡蛋只有一层蛋壳, 容易破裂。针对这个问题, 可以通过缩短酸刻蚀的时间、增加 反应物的浓度来对壳层厚度进行调控。MIL-101-Cr心想这下出征可算是十拿九稳, 已经拿出最喜欢 的俄罗斯套娃开始放松心情了。只听到他刚拆开第一个的时侯, 便突然自言自语起来: “如果在空 心结构的基础上继续生长MOFs, 那么壳层的厚度不照样可以增加了吗? 并且新生长出的MOFs仍存 在内外层的稳定性差异, 假如继续用酸刻蚀的话, 得到双层空心结构, 吸附容量还会增加! ” MIL101-Cr立即将这新奇的思路画了下来(图1)并潜心修炼。最后在吸附液相中布洛芬时, 身带三层空心 结构, 对布洛芬的吸附量达到了 $2000 \mathrm{mg} \cdot \mathrm{g}^{-1}[11]$ 。相对于实心结构的MIL-101-Cr, 吸附容量提升了近 一倍。众门派对MIL-101-Cr纷纷赞不绝口, 也明白了苦其心志和劳其筋骨的含义, 即利用酸刻蚀不 稳定的骨架结构, 得到空心材料。

在出征前的动员大会上, UiO-66早已立下投名状, 要带头处理废水中的染料大分子。可是转念 


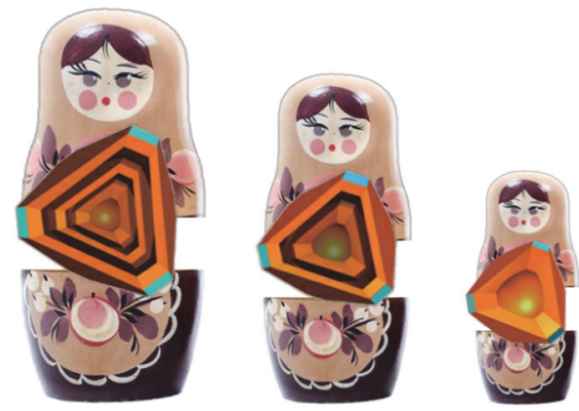

图1 俄罗斯套娃与MIL-101-Cr的多层空心结构[11]

一想, 自身孔道需要和吸附质分子直径相近才能有效吸附, 可自己是典型的微孔型材料, 这不是搬 起石头砸自己的脚嘛! UiO-66冷静下来, 开始苦心钻研军师所说的话, 领悟到了 “饿其体肤, 空乏 其身” 的奥妙之处。UiO-66认为其深层含义是指部分金属中心在混合配体中找不到对苯二甲酸进行 配位, 无奈之下只能选择与对苯二甲酸具有相同配位方式的单齿羧酸。这就相当于第三者插足, 第 三者的感情基础必然没有夫妻之间稳固, 在外界的干扰下也很容易被拆散。所以, 在酸处理之后, 不稳定的配体被选择性刻蚀, UiO-66内部产生配体空缺缺陷。这种缺陷的形成帮助UiO-66扩大了孔 径, 这下吸附废水中的染料应该是不在话下了。只见UiO-66纵身一跃, 经过一天一夜后, 成功将藏 红T溶液从红色转为无色 ${ }^{[12]}$ 。但是UiO-66却因为吸附了藏红 $T$, 整个身体都变成了红色, 这可把众人 吓坏了。其实吸星大法也存在缺点, 虽然吸收的内力会储存在自身孔洞中, 但是有异质反噬之险。 并且如果想继续使用, 必须把之前吸收的内力排出体外。UiO-66笑着说: “大家不必惊慌, 等我泡 个乙醇澡, 坐上旋转木马后再来收服剩下的敌人! ” UiO-66轻轻松松地就将吸附的异质排出了体外, 让众人惊叹, 并且经过数次循环再生后, 吸附容量并没有明显的降低。

\section{5 结语}

听了那么多关于MOFs的故事, 相信大家对这个新兴的门派也有了一定的了解, 让我们一起与行 侠仗义的MOFs在科技进步的道路上携手同行吧！

\section{参 考 文 献}

[1] Li, H.; Eddaoudi, M.; O’keeffe, M.; Yaghi, O. M. Nature 1999, 402 (6759), 276.

[2] Cavka, J. H.; Jakobsen, S.; Olsbye, U.; Guillou, N.; Lamberti, C.; Bordiga, S.; Lillerud, K. P. J. Am. Chem. Soc. 2008, 130 (42), 13850.

[3] Furukawa, H.; Cordova, K. E.; O’Keeffe, M.; Yaghi, O. M. Science 2013, 341 (6149), 123044.

[4] Férey, G.; Mellot-Draznieks, C.; Serre, C.; Millange, F.; Dutour, J.; Surblé, S.; Margiolaki, I. Science 2005,309 (5743), 2040.

[5] Park, K. S.; Ni, Z.; Côté, A. P.; Choi, J. Y.; Huang, R.; Uribe-Romo, F. J.; Chae, H. K.; O’Keeffe, M.; Yaghi, O. M. P. Natl. Acad. Sci. USA 2006, 103 (27), 10186.

[6] Chui, S. S. Y.; Lo, S. M. F.; Charmant, J. P. H.; Orpen, A. G.; Williams, I. D. Science 1999, 283 (5405), 1148.

[7] Farha, O. K.; Eryazici, I.; Jeong, N. C.; Hauser, B. G.; Wilmer, C. E.; Sarjeant, A. A.; Snurr, R. Q.; Nguyen, S. T.; Yazaydin, A. Ö.; Hupp, J. T. J. Am. Chem. Soc. 2012, 134 (36), 15016.

[8] Rosi, N. L.; Eckert, J.; Eddaoudi, M.; Vodak, D. T.; Kim, J.; O’Keefe, M.; Yaghi, O. M. Science 2003, 300 (5622), 1127.

[9] Tibbetts, G. G.; Meisner, G. P.; Olk, C. H. Carbon 2001, 39 (15), 2291.

[10] Jia, M.; Feng, Y.; Qiu, J.; Zhang, X. F.; Yao, J. Sep. Purif. Technol. 2019, 213, 63.

[11] Liu, W.; Huang, J.; Yang, Q.; Wang, S.; Sun, X.; Zhang, W.; Liu, J.; Huo, F. Angew. Chem. Int. Ed. 2017,129 (20), 5512.

[12] Wang, K.; Li, C.; Liang, Y.; Han, T.; Huang, H.; Yang, Q.; Liu, D.; Zhong, C. Chem. Eng. J. 2016, $289,486$. 